\title{
Common Fixed Points of Self Maps Satisfying an Integral Type Contractive Condition in Intuionistic Fuzzy Metric Space
}

\author{
Saurabh Manro \\ School of Mathematics and Computer Applications, Thapar University, \\ Patiala (Punjab) \\ Email: sauravmanro@yahoo.com
}

\begin{abstract}
In this paper, we prove two common fixed point theorems. In first theorem, we prove common fixed point theorem for two weakly compatible self maps of type (A) satisfying an integral type contractive condition in intuitionistic fuzzy metric space. In the second theorem, we prove common fixed point theorem for two weakly compatible maps satisfying an integral type contractive condition in intuitionistic fuzzy metric space. These results are proved without exploiting the notion of continuity and without imposing any condition of $t$-norm and $t$-conorm.
\end{abstract}

Index Terms - Intuitionistic fuzzy metric space, weakly compatible maps, weakly compatible maps of type (A), common fixed point

\section{INTRODUCTION}

Atanassove[1] introduced and studied the concept of intuitionistic fuzzy sets as a generalization of fuzzy sets. In 2004, Park[2] defined the notion of intuitionistic fuzzy metric space with the help of continuous $t$-norms and continuous $t$-conorms. Recently, in 2006, Alaca et al.[3] using the idea of Intuitionistic fuzzy sets, defined the notion of intuitionistic fuzzy metric space with the help of continuous $t$-norm and continuous $t$-conorms as a generalization of fuzzy metric space due to Kramosil and Michalek[4] . In 2006, Turkoglu[5] proved Jungck's[6] common fixed point theorem in the setting of intuitionistic fuzzy metric spaces for commuting mappings. Afterwards, many authors proved common fixed point theorems using different variants in such spaces. In this paper, we prove two common fixed point theorems. In first theorem, we prove common fixed point theorem for two weakly compatible self maps of type (A) satisfying an integral type contractive condition in intuitionistic fuzzy metric space. In the second theorem, we prove common fixed point theorem for two weakly compatible maps satisfying an integral type contractive condition in intuitionistic fuzzy metric space. These results are proved without exploiting the notion of continuity and without imposing any condition of $t$-norm and $t$-conorm.

\section{Preliminaries}

The concepts of triangular norms ( $t$-norm) and triangular conorms ( $t$-conorm) are known as the axiomatic skelton that we use are characterization fuzzy intersections and union respectively. These concepts were originally introduced by Menger in study of statistical metric spaces.

Definition 2.1[7]: A binary operation * : [0,1] $\times[0,1] \rightarrow$ $[0,1]$ is continuous $t$-norm if $*$ is satisfies the following conditions:

(i) * is commutative and associative;

(ii) $*$ is continuous;

(iii) $a * 1=a$ for all $a \in[0,1]$;

(iv) $a * b \leq c * d$ whenever $a \leq c$ and $b \leq d$ for all $a$, $b, c, d \in[0,1]$. 
Definition 2.2[7]: A binary operation $\diamond:[0,1] \times[0,1] \rightarrow$ $[0,1]$ is continuous $t$-conorm if $\diamond$ is satisfies the following conditions:

(i) $\diamond$ is commutative and associative;

(ii) $\diamond$ is continuous;

(iii) $a \diamond 0=a$ for all $a \in[0,1]$;

(iv) $a \diamond b \geq c \diamond \mathrm{d}$ whenever $a \leq c$ and $b \leq d$ for all $a, b$, $c, d \in[0,1]$.

Alaca et al.[3] defined the notion of intuitionistic fuzzy metric space as follows :

Definition 2.3[3]: A 5-tuple $(X, M, N, *, \diamond)$ is said to be an intuitionistic fuzzy metric space if $X$ is an arbitrary set, $*$ is a continuous $t$-norm, $\diamond$ is a continuous $t$-conorm and $M, N$ are fuzzy sets on $X^{2} \times[0, \infty)$ satisfying the following conditions:

(i) $M(x, y, t)+N(x, y, t) \leq 1$ for all $x, y$ in $X$ and $t>0$;

(ii) $M(x, y, 0)=0$ for all $x, y$ in $X$;

(iii) $M(x, y, t)=1$ for all $x, y$ in $X$ and $t>0$ if and only if $x=y$;

(iv) $\mathrm{M}(\mathrm{x}, \mathrm{y}, \mathrm{t})=\mathrm{M}(\mathrm{y}, \mathrm{x}, \mathrm{t})$ for all $x, y$ in $X$ and $t>0$;

(v) $\mathrm{M}(\mathrm{x}, \mathrm{y}, \mathrm{t}) * \mathrm{M}(\mathrm{y}, \mathrm{z}, \mathrm{s}) \leq \mathrm{M}(\mathrm{x}, \mathrm{z}, \mathrm{t}+\mathrm{s})$ for all $x, y, z$ in $X$ and $s, t>0$;

(vi) for all $x, y$ in $X, M(x, y,):.[0, \infty) \rightarrow[0,1]$ is left continuous;

(vii) $\lim _{t \rightarrow \infty} M(x, y, t)=1$ for all $x, y$ in $X$ and $t>0$;

(viii) $N(x, y, 0)=1$ for all $x, y$ in $X$;

(ix) $N(x, y, t)=0$ for all $x, y$ in $X$ and $t>0$ if and only if $x=y$;

(x) $N(x, y, t)=N(y, x, t)$ for all $x, y$ in $X$ and $t>0$;

(xi) $N(x, y, t) \diamond N(y, z, s) \geq N(x, z, t+s)$ for all $x, y, z$ in $X$ and $s, t>0$;

(xii) for all $x, y$ in $X, N(x, y,):.[0, \infty) \rightarrow[0,1]$ is right continuous;

(xiii) $\lim _{t \rightarrow \infty} N(x, y, t)=0$ for all $x, y$ in $X$ :

Then $(M, N)$ is called an intuitionistic fuzzy metric on $X$. The functions $M(x, y, t)$ and $N(x, y, t)$ denote the degree of nearness and the degree of non-nearness between $x$ and $y$ w.r.t. $t$ respectively.

Remark 2.4:[3] Every fuzzy metric space $(X, M, *)$ is an intuitionistic fuzzy metric space of the form
$(X, M, 1-M, *, \diamond)$ such that $t$-norm * and $t$-conorm $\diamond$ are associated as $x \diamond y=1-((1-x) *(1-y))$ for all $x, y$ in $X$.

Lemma 2.5:[3] In intuitionistic fuzzy metric space $(X, M, N, *, \diamond), M(x, y, *)$ is non-decreasing and $N(x, y, \diamond)$ is non-increasing for all $x, y$ in $X$.

Proof: Suppose that $M(x, y, *)$ is non-increasing, therefore for $t \leq s$, we have

$M(x, y, t) \geq M(x, y, s)$.

For all $x, y, z$ in $X$, we have

$M(x, z, t+s) \geq M(x, y, t) * M(y, z, s)$.

In particular for $z=y$, we have

$M(x, y, t+s) \geq M(x, y, t) * M(y, y, s)$.

$M(x, y, t+s) \geq M(x, y, t) * 1=M(x, y, t)$, a contradiction, hence $M\left(x, y,{ }^{*}\right)$ is non-decreasing.

Again, suppose $N(x, y, \diamond)$ is non-decreasing, therefore for $t \leq s$, we have

$N(x, y, s) \geq N(x, y, t)$.

For all $x, y, z$ in $X$, we have

$N(x, z, t+s) \leq N(x, y, t) \diamond N(y, z, s)$.

In particular for $z=y$, we have

$N(x, y, t+s) \leq N(x, y, t) \diamond N(y, y, s)$

$N(x, y, t+s) \leq N(x, y, t) \diamond 0=N(x, y, t)$, a contradiction, hence $N(x, y, \diamond)$ is non-increasing.

Definition 2.6[3]: Let $(X, M, N, *, \diamond)$ be an intuitionistic fuzzy metric space. Then

(a) a sequence $\left\{x_{n}\right\}$ in $X$ is said to be Cauchy sequence if, for all $t>0$ and $p>0$,

$$
\begin{aligned}
& \lim _{n \rightarrow \infty} M\left(x_{n+p}, x_{n}, t\right)=1 \\
& \text { and } \lim _{n \rightarrow \infty} N\left(x_{n+p}, x_{n}, t\right)=0 .
\end{aligned}
$$

(b) a sequence $\left\{\mathrm{x}_{\mathrm{n}}\right\}$ in $\mathrm{X}$ is said to be convergent to a point $x$ in $X$ if, for all $t>0$,

$$
\begin{aligned}
& \lim _{n \rightarrow \infty} M\left(x_{n}, x, t\right)=1 \\
& \text { and } \lim _{n \rightarrow \infty} N\left(x_{n}, x, t\right)=0 .
\end{aligned}
$$

Definition 2.7[3]: An intuitionistic fuzzy metric space $(X, M, N, *, \diamond)$ is said to be complete if and only if every Cauchy sequence in $X$ is convergent.

Example 2.8:[3] $X=\left\{\frac{1}{n}, n \in N\right\} \cup\{0\}$ and let * be the continuous $t$-norm and $\diamond$ be the continuous $t$ conorm defined by $a * b=a b$ and $a \diamond b=\min \{1, a+b\}$ 
respectively, for all $a, b \in[0,1]$. For each $t \in(0, \infty)$ and $x, y \in X$, define $(M, N)$ by

$$
\begin{aligned}
M(x, y, t) & =\left\{\begin{array}{cl}
\frac{\mathrm{t}}{\mathrm{t}+|\mathrm{x}-\mathrm{y}|}, & \mathrm{t}>0, \\
0 & \mathrm{t}=0
\end{array}\right. \\
\text { and } \quad & N(x, y, t)=\left\{\begin{array}{cl}
\frac{|\mathrm{x}-\mathrm{y}|}{\mathrm{t}+|\mathrm{x}-\mathrm{y}|}, & \mathrm{t}>0, \\
1 & \mathrm{t}=0
\end{array}\right.
\end{aligned}
$$

Clearly, $(X, M, N, *, \diamond)$ is complete intuitionistic fuzzy metric space.

Definition 2.9[5]: A pair of self mappings $(f, g)$ of a intuitionistic fuzzy metric space $(X, M, N, *, \diamond)$ is said to be commuting if

$$
\begin{aligned}
& M(f g x, g f x, t)=1 \text { and } \\
& N(f g x, g f x, t)=0 \text { for all } x \in X .
\end{aligned}
$$

Definition 2.10[5]: A pair of self mappings $(f, g)$ of a intuitionistic fuzzy metric space $(X, M, N, *, \diamond)$ is said to be weakly commuting if

$M(f g x, g f x, t) \geq M(f x, g x, t)$ and

$N(f g x, g f x, t) \leq N(f x, g x, t)$

for all $x \in X$ and $t>0$.

Definition 2.11[5]: A pair of self mappings ( $f, g)$ of a intuitionistic fuzzy metric space $(X, M, N, * \diamond) \quad$ is said to be compatible if

$$
\begin{aligned}
& \lim _{n \rightarrow \infty} M\left(f g x_{n}, g f x_{n}, t\right)=1, \\
& \lim _{n \rightarrow \infty} N\left(f g x_{n}, g f x_{n}, t\right)=0
\end{aligned}
$$

for all $t>0$, whenever $\left\{x_{n}\right\}$ is a sequence in $X$ such that $\lim _{n \rightarrow \infty} f x_{n}=\lim _{n \rightarrow \infty} g x_{n}=u$ for some $u$ in $X$.

Definition 2.12 [8]: Two self maps $f$ and $g$ on a set $X$ are said to be weakly compatible if they commute at the coincidence points i.e., if $f u=g u$ for some $u$ in $X$, then $f g u=g f u$.

It is easy to see that two compatible maps are weakly compatible but converse is not true.

Definition 2.13: A pair of self mappings $(f, g)$ of a intuitionistic fuzzy metric space $(X, M, N, *, \diamond)$ is said to be weakly compatible of type $(A)$ if

$$
\begin{aligned}
& \lim _{n \rightarrow \infty} M\left(f g x_{n}, g g x_{n}, t\right)=1, \\
& \lim _{n \rightarrow \infty} N\left(f g x_{n}, g g x_{n}, t\right)=0 \\
& o r \\
& \lim _{n \rightarrow \infty} M\left(g f x_{n}, f f x_{n}, t\right)=1, \\
& \lim _{n \rightarrow \infty} N\left(g f x_{n}, f f x_{n}, t\right)=0
\end{aligned}
$$

for all $t>0$, whenever $\left\{x_{n}\right\}$ is a sequence in $X$ such that $\lim _{n \rightarrow \infty} f x_{n}=\lim _{n \rightarrow \infty} g x_{n}=u$ for some $u$ in $X$.

\section{MAIN RESUlt}

Now, we prove our main theorem as follows:

Theorem 3.1: Let $f$ and $g$ be weakly compatible maps of type $(A)$ of a complete intuitionistic fuzzy metric space

$(X, M, N, *, \diamond)$ satisfying the following conditions:

(3.1) $\mathrm{f}(\mathrm{X}) \subseteq \mathrm{g}(\mathrm{X})$

(3.2) any one of the mappings for $g$ is continuous

$$
\begin{aligned}
\int_{0}^{1-M(f x, f y, c t)} \varphi(t) d t & <\int_{0}^{1-M(g x, g y, t)} \varphi(t) d t, \\
\int_{0}^{1-N(f x, f y, c t)} \varphi(t) d t & >\int_{0}^{1-N(g x, g y, t)} \varphi(t) d t
\end{aligned}
$$

for each $\mathrm{x}, \mathrm{y}$ in $\mathrm{X}, \mathrm{t}>0, c \in[0,1)$, where $\varphi: \mathrm{R}^{+} \rightarrow \mathrm{R}$ is a lebesgue integrable mapping which is summable, non negative and such that

(3.4) $\int_{0}^{\epsilon} \varphi(t) d t>0$ for each $\in>0$.

Then $f$ and $g$ have a unique common fixed point.

Proof: Let $x_{o} \in X$. Since $f(X) \subseteq g(X)$, choose $x_{1} \in X$ such that $g x_{1}=f x_{0}$. Choose $x_{n+1}$ such that $y_{n}=g x_{n+1}=f x_{n}$. We now show that $\left\{y_{n}\right\}$ is a Cauchy sequence. For each integer $n \geq 1$, and from (3.3), we have

$$
\begin{aligned}
\int_{0}^{1-M\left(y_{n}, y_{n+1}, t\right)} \varphi(t) d t & =\int_{0}^{1-M\left(f x_{n}, f x_{n+1}, t\right)} \varphi(t) d t \\
& <\int_{0}^{1-M\left(g x_{n}, g x_{n+1}, \frac{t}{c}\right)} \varphi(t) d t \\
& =\int_{0}^{1-M\left(f x_{n-1}, f x_{n}, \frac{t}{c}\right)} \varphi(t) d t \\
& <\int_{0}^{1-M\left(g x_{n-1}, g x_{n}, \frac{t}{c^{2}}\right)} \varphi(t) d t
\end{aligned}
$$


and

$$
\begin{aligned}
\int_{0}^{1-N\left(y_{n}, y_{n+1}, t\right)} \varphi(t) d t & =\int_{0}^{1-N\left(f x_{n}, f x_{n+1}, t\right)} \varphi(t) d t \\
& >\int_{0}^{1-N\left(g x_{n}, g x_{n+1}, \frac{t}{c}\right)} \varphi(t) d t \\
& =\int_{0}^{1-N\left(f x_{n-1}, f x_{n}, \frac{t}{c}\right)} \varphi(t) d t \\
& >\int_{0}^{1-N\left(g x_{n-1}, g x_{n}, \frac{t}{c^{2}}\right)} \varphi(t) d t .
\end{aligned}
$$

In this fashion, we obtain

$$
\begin{aligned}
& \int_{0}^{1-M\left(y_{n}, y_{n+1}, t\right)} \varphi(t) d t<\int_{0}^{1-M\left(y_{0}, y_{1}, \frac{t}{c^{n}}\right)} \varphi(t) d t \\
& \text { and } \\
& \int_{0}^{1-N\left(y_{n}, y_{n+1}, t\right)} \varphi(t) d t>\int_{0}^{1-N\left(y_{0}, y_{1}, \frac{t}{c^{n}}\right)} \varphi(t) d t .
\end{aligned}
$$

Now letting $n \rightarrow \infty$ and using Lebesgue dominated convergence theorem, it follows in view of (3.4),

$$
\begin{aligned}
& \lim _{n \rightarrow \infty} M\left(y_{n}, y_{n+1}, t\right)=1 \\
& \lim _{n \rightarrow \infty} N\left(y_{n}, y_{n+1}, t\right)=0
\end{aligned}
$$

Similarly,

$$
\begin{aligned}
& \lim _{n \rightarrow \infty} M\left(y_{n+1}, y_{n+2}, t\right)=1 \\
& \lim _{n \rightarrow \infty} N\left(y_{n+1}, y_{n+2}, t\right)=0
\end{aligned}
$$

Now for any positive integer $p$, we have

$$
\begin{aligned}
& M\left(y_{n}, y_{n+p}, t\right) \geq M\left(y_{n}, y_{n+1}, \frac{t}{p}\right) * . . \\
& \qquad . p-\operatorname{times} \ldots * M\left(y_{n+p-1}, y_{n+p}, \frac{t}{p}\right) \\
& \geq M\left(y_{n}, y_{n+1}, \frac{t}{p}\right) * \ldots p-\operatorname{times} \ldots * M\left(y_{n}, y_{n+1}, \frac{t}{p}\right) \\
& \text { and } \\
& N\left(y_{n}, y_{n+p}, t\right) \leq N\left(y_{n}, y_{n+1}, \frac{t}{p}\right) \diamond . . \\
& \quad . p-\operatorname{times} \ldots \diamond N\left(y_{n+p-1}, y_{n+p}, \frac{t}{p}\right) \\
& \leq N\left(y_{n}, y_{n+1}, \frac{t}{p}\right) \diamond \ldots p-\operatorname{times} \ldots \diamond N\left(y_{n}, y_{n+1}, \frac{t}{p}\right)
\end{aligned}
$$

\section{Since}

$\lim _{n \rightarrow \infty} M\left(y_{n}, y_{n+1}, t\right)=1$
and
$\lim _{n \rightarrow \infty} N\left(y_{n}, y_{n+1}, t\right)=0$,

It follows that

$\lim _{n \rightarrow \infty} M\left(y_{n}, y_{n+p}, t\right) \geq 1 * 1 * \ldots * 1 \geq 1$

and

$\lim _{n \rightarrow \infty} N\left(y_{n}, y_{n+1}, t\right) \leq 0 \diamond 0 \diamond \ldots \diamond 0 \leq 0$.

Thus $\left\{y_{n}\right\}$ is a Cauchy sequence in $X$. Since $X$ is complete, so there exist a point $z$ in $g(X)$ such that $\lim _{n \rightarrow \infty} y_{n}=z$.

Hence $\lim _{n \rightarrow \infty} g x_{n+1}=\lim _{n \rightarrow \infty} f x_{n}=z$. Since either $f$ or $g$ is continuous, let $g$ be continuous, therefore $\lim _{n \rightarrow \infty} g f x_{n}=g z$.

But $f$ and $g$ are weakly compatible of type $(A)$, $\lim _{n \rightarrow \infty} f g x_{n}=g z$.

Now from (3.3), we have

$$
\begin{aligned}
& \int_{0}^{1-M\left(f g x_{n}, f x_{n}, t\right)} \varphi(t) d t<\int_{0}^{1-M\left(g g x_{n}, g x_{n}, \frac{t}{c}\right)} \varphi(t) d t \\
& \text { and } \\
& \int_{0}^{1-N\left(f g x_{n}, f x_{n}, t\right)} \varphi(t) d t>\int_{0}^{1-N\left(g g x_{n}, g x_{n}, \frac{t}{c}\right)} \varphi(t) d t .
\end{aligned}
$$

Letting $n \rightarrow \infty$ and using Lebesgue dominated convergence theorem, it follows in view of (3.4), that $g z=z$.

Again from (3.3), we have

$$
\begin{aligned}
& \int_{0}^{1-M\left(f x_{n}, f z, t\right)} \varphi(t) d t<\int_{0}^{1-M\left(g x_{n}, g z, \frac{t}{c}\right)} \varphi(t) d t \\
& \text { and } \\
& \int_{0}^{1-N\left(f x_{n}, f z, t\right)} \varphi(t) d t>\int_{0}^{1-N\left(g x_{n}, g z, \frac{t}{c}\right)} \varphi(t) d t .
\end{aligned}
$$

Taking $n \rightarrow \infty$ and using Lebesgue dominated convergence theorem, it follows in view of (3.4), that $f z=$ $z$. Hence, $z$ is a common fixed point of $f$ and $g$.

For Uniqueness: Suppose that $w(\neq z)$ is also another fixed point of $f$ and $g$. Then, we have 


$$
\begin{aligned}
\int_{0}^{1-M(z, w, t)} \varphi(t) d t & =\int_{0}^{1-M(f z, f w, t)} \varphi(t) d t \\
& <\int_{0}^{1-M\left(g z, g w, \frac{t}{c}\right)} \varphi(t) d t \\
& =\int_{0}^{1-M\left(z, w, \frac{t}{c}\right)} \varphi(t) d t
\end{aligned}
$$

and

$$
\begin{aligned}
\int_{0}^{1-N(z, w, t)} \varphi(t) d t & =\int_{0}^{1-N(f z, f w, t)} \varphi(t) d t \\
& >\int_{0}^{1-N\left(g z, g w,-\frac{t}{c}\right)} \varphi(t) d t \\
& =\int_{0}^{1-N\left(z, w, \frac{t}{c}\right)} \varphi(t) d t
\end{aligned}
$$

where $c \in[0,1)$, which implies $z=w$ and so uniqueness follows.

Theorem 3.2: Let $f$ and $g$ be weakly compatible self maps of intuitionistic fuzzy metric space $(X, M, N, *, \diamond)$ satisfying (3.1), (3.3), (3.4) and the following conditions: (3.6) any one of $f(X)$ or $g(X)$ is closed subset of $X$

Then $f$ and $g$ have a unique common fixed point.

Proof: From proof of above theorem, we conclude that $\left\{y_{n}\right\}$ is a Cauchy sequence in $X$ and since either $f(X)$ or $g(X)$ is closed, for definiteness assume that $g(X)$ is closed subset of $X$. Note that sequence $\left\{y_{2 n}\right\}$ is contained in $g(X)$ and has a limit in $g(X)$, call it $z$. Let $u$ in $g^{-1} z$. Then $g u=z$. Now we show that $f u=z$. From (3.3), we have

$$
\begin{aligned}
& \int_{0}^{1-M\left(f x_{n}, f u, t\right)} \varphi(t) d t<\int_{0}^{1-M\left(g x_{n}, g u, \frac{t}{c}\right)} \varphi(t) d t \\
& \text { and } \\
& \int_{0}^{1-N\left(f x_{n}, f u, t\right)} \varphi(t) d t>\int_{0}^{1-N\left(g x_{n}, g u, \frac{t}{c}\right)} \varphi(t) d t
\end{aligned}
$$

Taking $n \rightarrow \infty$ and using Lebesgue dominated convergence theorem, it follows in view of (3.4), that $f u=z$.

Since $f$ and $g$ are weakly compatible, therefore, it follow that $f z=f g u=g f u=g z$.

Now, we show that $z$ is a common fixed point of $f$ and g. From (3.3), we have

$$
\begin{aligned}
\int_{0}^{1-M(f z, z, t)} \varphi(t) d t & =\int_{0}^{1-M(f z, f u, t)} \varphi(t) d t \\
& <\int_{0}^{1-M\left(g z, g u, \frac{t}{c}\right)} \varphi(t) d t \\
& =\int_{0}^{1-M\left(g z, g u, \frac{t}{c}\right)} \varphi(t) d t
\end{aligned}
$$

and

$$
\begin{aligned}
\int_{0}^{1-N(f z, z, t)} \varphi(t) d t & =\int_{0}^{1-N(f z, f u, t)} \varphi(t) d t \\
& >\int_{0}^{1-N\left(f z, z, \frac{t}{c}\right)} \varphi(t) d t \\
& =\int_{0}^{1-N\left(f z, z, \frac{t}{c}\right)} \varphi(t) d t
\end{aligned}
$$

which is a contradiction, since $c \in[0,1)$, therefore, $f z=z=g z$ and hence, $z$ is a common fixed point of $f$ and $g$.

For Uniqueness: Suppose that $w(\neq z)$ is also another fixed point of $f$ and $g$. Then, we have

$$
\begin{aligned}
\int_{0}^{1-M(z, w, t)} \varphi(t) d t & =\int_{0}^{1-M(f z, f w, t)} \varphi(t) d t \\
& <\int_{0}^{1-M\left(g z, g w, \frac{t}{c}\right)} \varphi(t) d t \\
& =\int_{0}^{1-M\left(z, w, \frac{t}{c}\right)} \varphi(t) d t
\end{aligned}
$$

and

$$
\begin{aligned}
\int_{0}^{1-N(z, w, t)} \varphi(t) d t & =\int_{0}^{1-N(f z, f w, t)} \varphi(t) d t \\
& >\int_{0}^{1-N\left(g z, g w, \frac{t}{c}\right)} \varphi(t) d t \\
& =\int_{0}^{1-N\left(g z, g w, \frac{t}{c}\right)} \varphi(t) d t
\end{aligned}
$$

where $c \in[0,1)$, which implies $z=w$ and so uniqueness follows.

Example 3.3: Let $\mathrm{X}=[0,1]$. Define

$$
M(x, y, t)=\left\{\begin{array}{cc}
\frac{\mathrm{t}}{\mathrm{t}+|\mathrm{x}-\mathrm{y}|}, & \mathrm{t}>0, \\
0 & \mathrm{t}=0
\end{array}\right.
$$


and

$$
N(x, y, t)=\left\{\begin{array}{cc}
\frac{|\mathrm{x}-\mathrm{y}|}{\mathrm{t}+|\mathrm{x}-\mathrm{y}|}, & \mathrm{t}>0, \\
1 & \mathrm{t}=0
\end{array}\right.
$$

for all $x, y$ in $X$ and for each $t>0$. Define self mappings $f$ and $g$ on $X$ by $f(x)=\frac{x}{3}, g(x)=\frac{x}{2}$ for all $x$ in $X$. Let $\varphi(t)=t$ for $t>0$. Clearly all conditions of theorem 3.1 satisfied for all $c \in\left[\frac{2}{3}, 1\right)$ and $x=0$ is the unique common fixed point of $f$ and $g$.

\section{ACKNOWLEDGMENT}

The author wish to thank Dr Sanjay Kumar, Dr S. S. Bhatia, Dr. Sunny Chauhan, Dr Suneel Kumar and the referees for their very helpful suggestions and many kind comments.

\section{REFERENCES}

[1] K. Atanassov, Intuitionistic Fuzzy sets, Fuzzy sets and system, 20, 1986, pp. 87-96.

[2] J. H. Park, Intuitionistic fuzzy metric spaces, Chaos, Solitons \& Fractals, 22, 2004 pp. 1039- 1046.

[3] C. Alaca, D. Turkoglu, and C. Yildiz, Fixed points in Intuitionistic fuzzy metric spaces, Chaos, Solitons \& Fractals, 29, 2006, pp. 1073-1078.

[4] I. Kramosil and J. Michalek, Fuzzy metric and Statistical metric spaces, Kybernetica, 11, 1975, pp. 326-334.

[5] D. Turkoglu, C. Alaca and C. Yildiz, Compatible maps and Compatible maps of type $(\alpha)$ and $(\beta)$ in intuitionistic fuzzy metric spaces, Demonstratio Math., 39, No.3, 2006, pp. 671-684.

[6] G. Jungck, Commuting mappings and fixed points, Amer. Math. Monthly, 83,1976, pp. 261-263.

[7] B. Schweizer and A. Sklar, Probabilistic Metric Spaces, Morth Holland Amsterdam,1983.

[8] G. Jungck, BE Rhoades, Fixed point for set valued functions without continuity, Indian J Pure Appl Math, 29, No.3, 1998, pp. 227-238.
Saurabh Manro is the Research Scholar in School of Mathematics and Computer Applications, Thapar University, Patiala (Punjab), India. He completed M.Sc (Mathematics) in year 2004 from Punjab University, Chandigarh. He is pursuing Ph.D under the kind supervision of Dr. S.S. Bhatia and Dr. Sanjay Kumar. His areas of research include Fixed point theorem in various abstract spaces like Menger spaces, Probabilistic Metric spaces, Fuzzy Metric spaces, Intuitionistic Fuzzy Metric spaces, G- Metric spaces and its applications. He has published many research papers in national / international papers till now. Some papers are ready to be published. 